Lengua y Sociedad. Revista de Lingüística Teórica y Aplicada Vol. 20, n. ${ }^{\circ}$ 1, Lima, enero-junio de 2021, pp. 101-124 https://doi.org/10.15381/lengsoc.v20i1.22271

\title{
Documentación lingüística-cultural y producción de prototipos de material educativo para el desarrollo de programas de fortalecimiento $y$ revitalización del quechua en asentamientos humanos del distrito de Los Olivos-Lima ${ }^{1}$
}

\section{Linguistic-cultural documentation and production of prototypes of educational material for the development of programs to strengthen and revitalize Quechua in human settlements in the Los Olivos-Lima district}

\author{
Carmela Florencia López Capillo \\ Universidad Nacional Federico Villarreal, Lima, \\ Perú \\ Código Orcid: 0ooo-0oo2-5414-1699 \\ clopezc@unfv.edu.pe
}

\author{
Yris Barraza de García \\ ISP Formabiap Iquitos, Perú \\ Código Orcid: oooo-0oo3-2975-1927 \\ ybarraza.dall@gmail.com
}

\author{
Rosalinn Cancino Verde \\ Universidad Nacional Federico Villarreal, Lima, Perú \\ Código Orcid: oooo-0003-0249-5345 \\ cancinoverdefrancisca@gmail.com
}

\begin{abstract}
Resumen
El artículo ofrece los resultados de un estudio de investigación que documentó discursos que representan los saberes lingüísticos y culturales de los hablantes de quechua de los grupos dialectales: Áncash-Huaylas-Huánuco-Lima, Huancavelica-Ayacucho-Ica y Apurímac-Cuzco-Puno, con el propósito de desarrollar materiales para la revitalización de la lengua originaria en los asentamientos humanos Enrique Milla Ochoa, Laura Caller Iberico y Armando Villanueva del Campo del distrito de Los Olivos de Lima, áreas urbanas en las que los hijos de los migrantes fundadores

Investigación auspiciada por la UNFV conforme lo establecido en la Resolución Rectoral $\mathrm{N}^{\circ} 7095$ 2020-CU-UNFV. Las responsables del estudio agradecen a los siguientes colaboradores por todo el apoyo prestado: Félix Julca Guerrero, Universidad Santiago Antúnez de Mayolo; Ancash; Helí Vidal López y Aída López Capillo, Pomabamba, Ancash; Fanel Guevara Guevara, Cusco; Egresados y Estudiantes Especialidad de Lingüística Unfv; Municipalidad Distrital de Los Olivos, y Autoridades AAHH Grupo Confraternidad - Los Olivos.
\end{abstract}


Documentación lingüística-cultural y producción de prototipos de material... Carmela Florencia López Capillo, Yris Barraza de García y Rosalinn Cancino Verde

no la hablan. Mediante el uso de la Internet y con la contribución de cultivadores del idioma, en un primer momento, se identificó, en distintos sitios web, recursos gráficos, audios y videos de interés; posteriormente, se reunió, clasificó, adecuó y tradujo textos literarios, recreativos e instructivos para producir materiales nuevos bajo parámetros específicos; asimismo, se elaboró guías para impulsar la transmisión natural de la lengua, la activación de habilidades expresivas en semilingües, nidos de lenguas polidialectales, talleres de lectura y escritura para hablantes fluidos de quechua y de aprendizaje de segunda lengua para monolingües de habla hispana. Los productos finales también serán utilizados en nuevos programas.

Palabras clave: revitalización lingüística, quechua en la ciudad, migración y quechua

\begin{abstract}
The article offers the results of a research study that documented speeches that represent the linguistic and cultural knowledge of Quechua speakers of the dialect groups: Ancash-HuaylasHuánuco-Lima, Huancavelica-Ayacucho-Ica and Apurimac-Cuzco-Puno, with the purpose of developing materials for the revitalization of the original language in the human settlements Enrique Milla Ochoa, Laura Caller Iberico and Armando Villanueva del Campo of the Los Olivos district of Lima, urban areas in which the children of the founding migrants do not they speak. Through the use of the Internet and with the contribution of language cultivators, at first, graphic resources, audios and videos of interest were identified on different websites; later, it was collected, classified, adapted and translated literary, recreational and instructive texts to produce new materials under specific parameters; likewise, guides were developed to promote the natural transmission of the language, the activation of expressive skills in semi-linguists, nests of polidialectal languages, reading and writing workshops for fluent Quechua speakers and second language learning for Spanish-speaking monolinguals. The final products will also be used in new programs.
\end{abstract}

Keywords: linguistic revitalization, Quechua in the city, migration and Quechua.

Publicado: I5-07-202I

\title{
1. Introducción
}

\subsection{Planteamiento del problema}

López, Sullcahuamán y Barraza (2019) y López y Barraza (2018) dan conocer la situación sociolingüística de la población migrante de Los Olivos (Organización Internacional para las Migraciones [OIM], 2015), uno de los distritos más jóvenes y dinámicos de Lima. Ambos estudios reportan la presencia de quechua hablantes de los grupos dialectales: Ancash-Huánuco-Lima-Junín, Huancavelica-Ayacucho-Ica y Apurímac-Cuzco-Puno, en los asentamientos humanos (AA. Hн.) Enrique Milla Ochoa, Laura Caller Iberico y Armando Villanueva del Campo de este distrito. 
Documentación lingüística-cultural y producción de prototipos de material... Carmela Florencia López Capillo, Yris Barraza de García y Rosalinn Cancino Verde

Con relación a los fundadores de dichos conglomerados habitacionales ${ }^{2}$, informan que desafortunadamente estos no han logrado transferir la lengua nativa a sus descendientes (Gutiérrez, 2013; Unesco, 2003); pues, la hegemonía del contexto limeño hispanohablante les impidió hacerlo. En la actualidad, sin embargo, desde el Estado y desde distintos organismos gubernamentales (Ley N. ${ }^{\circ} 29735$, Decreto Supremo N. ${ }^{\circ}$ o05-2017-MC y Ley Universitaria 30220), las lenguas originarias son valoradas, principalmente, como símbolos de identidad sociocultural y como vehículos de comunicación de diverso alcance. Debido a que esta nueva situación jurídica, los migrantes olivenses muestran interés por la revitalización de la lengua en sus comunidades. Dadas las circunstancias, corresponde implementar, programas de retención, fortalecimiento y aprendizaje de la lengua nativa; entre ellos, nidos de lengua naturales y comunitarios para establecer puentes entre abuelos y nietos ${ }^{3}$; talleres de lectura y escritura y de creación poética para empoderar a los migrantes fundadores y a quienes se incorporaron posteriormente; asimismo, talleres de aprendizaje de quechua como segunda lengua para los hijos de estos. Materializar tales programas demanda la previsión de una amplia gama de información lingüística y cultural, testimonios con los que los planificadores no contaban antes del inicio de la presente investigación.

Las preguntas concretas que originaron la pesquisa fueron las siguientes:

\subsubsection{Cuestión General}

¿Qué saberes lingüísticos y culturales tradicionales poseen los pueblos quechuas, y evidentemente los migrantes que residen en Lima Metropolitana, qué discursos los expresan; cuáles de estos son adecuados para diseñar materiales de apoyo para la ejecución de programas de revitalización lingüística en el distrito de Los Olivos; y qué características deben tener aquellos?

2 El apurimeño Buenaventura Usaqui refiere que él y otros migrantes del interior del país fundaron el conglomerado habitacional Confraternidad integrado por nueve asentamientos humanos; entre ellos, Laura Caller Iberico (donde él reside), Enrique Milla Ochoa y Armando Villanueva del Campo, en 1989.

3 Los hijos de migrantes fundadores no hablan quechua; en el mejor de los casos, son semilingües; por tanto, el programa de transmisión tendrá como protagonistas a los padres e hijos de estos; abuelos y nietos, en la cadena de sucesión. 
Documentación lingüística-cultural y producción de prototipos de material... Carmela Florencia López Capillo, Yris Barraza de García y Rosalinn Cancino Verde

\subsubsection{Cuestiones Específicas}

¿Qué saberes lingüísticos y culturales tradicionales poseen los pueblos quechuas, grupos a los cuales pertenecen los migrantes que residen en Lima Metropolitana, y discursos de qué géneros y especies los expresan?

Del conjunto de discursos tradicionales registrados, por la pertinencia social y cultural de sus contenidos, ¿cuáles son adecuados para diseñar materiales de apoyo para el desarrollo de programas de revitalización lingüística en los asentamientos humanos del distrito de Los Olivos?

¿Qué características tendrían los materiales de apoyo referidos de manera que sean valiosos para la ejecución de los programas de revitalización lingüística?

\subsection{Objetivos}

Con el propósito buscar respuestas para las preguntas anteriores, la pesquisa propuso los objetivos siguientes:

\subsubsection{General}

Documentar los discursos que los pueblos quechuas -entre ellos, los migrantes que residen en Lima Metropolitana- utilizan para expresan sus saberes lingüísticos y culturales; asimismo, del conjunto de estos textos registrados, según sus géneros y sus especies, seleccionar aquellos que son adecuados para diseñar materiales de apoyo para la revitalización del quechua en el distrito de Los Olivos; finalmente, proponer criterios técnicos básicos para desarrollar esta última tarea con relativo éxito.

\subsubsection{Específicos}

Identificar y registrar los diferentes discursos que expresan los saberes lingüísticos y culturales de los pueblos quechua, grupos a los cuales pertenecen los migrantes que residen en Lima Metropolitana; asimismo, clasificarlos según los géneros y subgéneros a los que pertenecen.

Del conjunto de discursos quechuas registrados, seleccionar aquellos que, debido a la pertinencia social y cultural de sus contenidos, son adecuados para diseñar materiales de apoyo para el desarrollo de programas de revitalización lingüística en los asentamientos humanos del distrito de Los Olivos de Lima.

Proponer criterios técnicos básicos para desarrollar materiales de apoyo para la ejecución de las distintas modalidades del programa referido.

104 Lengua y Sociedad. Revista de Lingüística Teórica y Aplicada 
Documentación lingüística-cultural y producción de prototipos de material... Carmela Florencia López Capillo, Yris Barraza de García y Rosalinn Cancino Verde

\subsection{Justificación e importancia}

La investigación permitió documentar muestras valiosas de saberes expresados en discursos en lengua quechua, conocimientos culturales y lingüísticos que los migrantes asentados en sectores urbanos y semiurbanos de Lima poseen también. Los textos orales y escritos inéditos contienen información base para la construcción de materiales físicos o digitales, -similares a los identificados en la Internetpara ser utilizados en la revitalización de variedades quechua amenazadas de desaparecer de los asentamientos humanos del distrito de Los Olivos, junto con los migrantes de primera generación que no pudieron dejarlas en herencia a sus descendientes. Asimismo, los corpus reunidos formarán parte del repositorio de lenguas originarias de acceso libre que la universidad de procedencia de las investigadoras implementa en la actualidad.

De otro lado, el estudio es socialmente relevante por el impacto favorable de las tareas preliminares de la revitalización lingüística en la población del distrito de Los Olivos. La certeza de que sus saberes serán plasmados en materiales de última generación les permite reconocerse como valiosos por sí mismos, fortalecer sus identidades sociolingüísticas y participar activamente en realización del proyecto.

\section{Antecedentes}

Existen diversos programas de documentación lingüística desarrollados en el contexto latinoamericano. México, igual que Perú, es un país multilingüe y pluricultural que ha acumulado importantes experiencias. El Instituto Nacional de Lenguas Indígenas (INALI, 2012), creado bajo el amparo de la Ley General de los Derechos Lingüísticos de los Pueblos Indígenas de 2003, da cuenta de la ejecución del proyecto denominado Documentación Lingüística. Este consistió en una serie de estudios que, como parte del Programa de Revitalización, Fortalecimiento y Desarrollo de las Lenguas Indígenas Nacionales 2008-2012 (PINALI), registró muestras de textos de lenguas en peligro de extinción. En la misma línea, Leyva (2014) comparte información específica respecto de la documentación de la lengua kumiai. El INALI (2012) refiere que se trata de un idioma integrante de la familia kochimí-yumana hablada en Baja California y amenazada de extinción por presión del español y de otros idiomas. Finalmente, Rodríguez y Magaña (2017) describen como casos de revitalización de culturas y lenguas, experiencias musicales que, en los últimos años, protagonizan jóvenes indígenas mexicanos. Estos fusionan ritmos propios y occidentales para motivar a quienes ya no desean utilizar las lenguas originarias. 
Documentación lingüística-cultural y producción de prototipos de material... Carmela Florencia López Capillo, Yris Barraza de García y Rosalinn Cancino Verde

Acerca de la documentación de lenguas originarias peruanas, existen publicaciones que informan respecto de los avances. Valqui y otros (2016) presentan los resultados de un proyecto colaborativo de acopio de textos orales en las lenguas: ashaninka, yanesha y nomatsiguenga. Entre los antecedentes de este trabajo, citan a los repositorios internacionales Endangered Languages Archive (ELAR) y Dokumentation Bedrohter Sprachen (DOBES) que, en conjunto, reúnen datos primarios provenientes de 15 lenguas amazónicas de afiliación: Arawak, Bora, Kandozi, Huitoto, Pano, Shimaco, Tupi-guarani y Zaparo, amenazadas o en peligro de extinción (Valqui y otros, 2016, pp. 64-66). Por su parte, el Archivo Digital de Lenguas Peruanas (ADLP) de la Pontificia Universidad Católica del Perú registra antologías de material audiovisual de 19 lenguas de afiliación pano, quechua, aimara, arawá, jíbaro (incluyendo la lengua de señas peruana, LSP). Finalmente, Zariquey y otros (2019) ofrecen un balance de los «logros alcanzados y los desafíos pendientes en materia de documentación y descripción de la diversidad lingüística peruana». Advierten la escasez de trabajos para el conocimiento científico de las lenguas indígenas y la ausencia de materiales para la revitalización de lenguas amenazadas y en peligro de extinción; para revertir la situación, demandan la participación del Estado peruano.

\section{Marco Teórico}

\subsection{Documentación lingüística}

El estudio desarrollado se inscribe en el ámbito de la documentación lingüística, sector de la investigación científica que, en términos de Himmelmann (1998), se encarga de acopiar muestras variadas de producción verbal en contextos reales de comunicación e incrementar dicho bagaje con testimonios confiables y comprensibles que faciliten su empleo en tareas de revitalización lingüística y de descripción científica, por parte de los pueblos que hablan las lenguas registradas o por parte de lingüistas, antropólogos, sociólogos y otros.

\subsection{Datos objetivo en la documentación lingüística}

Considerando que «una documentación lingüística es un registro duradero y multifuncional de una lengua». Hilmmelman (2007) sostiene que, para lograr un registro con estas características, se debe reunir datos primarios de una amplia gama de variedades (geográficas y sociales) y de géneros orales y escritos

106 Lengua y Sociedad. Revista de Lingüística Teórica y Aplicada 
Documentación lingüística-cultural y producción de prototipos de material... Carmela Florencia López Capillo, Yris Barraza de García y Rosalinn Cancino Verde

(narraciones, descripciones, conversaciones, instrucciones; poesías, canciones, metáforas cotidianas y expresiones idiosincrásicas; leyendas, mitos, adivinanzas) de la lengua objetivo; asimismo, cuidar la calidad de las grabaciones, transcripciones, glosas y metadatos.

\subsection{Los datos para la revitalización de lenguas}

Austin (2007), quien cita a Woodbury, recomienda que el conjunto de datos útiles para el mantenimiento y el aprendizaje de una lengua amenazada debe ser diverso, amplio, continuo, distribuido y flexible; transparente, preservable, portátil y ético.

\section{Metodología}

\subsection{Tipo de investigación}

La investigación fue desarrollada desde un enfoque cualitativo con firmes propósitos aplicativos. En tal sentido, documentó muestras de discursos que representan los saberes lingüísticos y culturales de los quechua hablantes del Perú para la ejecución de tareas sociales y culturales específicas.

Ámbitos espacial y temporal del estudio: Lima Metropolitana 2020.

Unidad de Análisis: Saberes lingüísticos y culturales tradicionales de quechua hablantes de las variedades registradas y agrupadas por López, Sullcahuamán y Barraza (2019) y López y Barraza (2018).

\subsection{Población y muestra}

La población estuvo conformada todos los hablantes quechua de las macro variedades Ancash-Huánuco-Lima-Junín, Huancavelica-Ayacucho-Ica y ApurímacCuzco-Puno.

La muestra estuvo constituida por mujeres y varones poseedores de información cultural abundante y/o con habilidades lingüísticas y artísticas especiales que representan a los quechua hablantes de las macro variedades precisadas en 3.1. La participación de un número significativo de colaboradores nacidos en Ancash se sustenta en los reportes del Instituto Nacional de Estadística e Informática ([INEI], 2018) y López, Sullcahuamán y Barraza (2019), quienes notaron la presencia mayoritaria de migrantes de esa región en Los Olivos, distrito beneficiario de los programas de revitalización. 
Documentación lingüística-cultural y producción de prototipos de material... Carmela Florencia López Capillo, Yris Barraza de García y Rosalinn Cancino Verde

\subsection{Instrumentos y Materiales}

El material lingüístico, parte del cual se muestra en la sección 5, consta de discursos en las variedades quechua precisadas en 3.2, de autoría, género y formato diverso. En cuanto a su origen, un grupo fue tomado de sitios web institucionales o particulares a los que se tuvo acceso a través de la Internet ${ }^{4}$; otro grupo fue creado o recreado (adaptado de otra variedad) de forma ad hoc para su uso en los programas de revitalización. Acerca de las autorías, los textos escritos, audios y videos pertenecen a artistas particulares o entidades colectivas. Según los géneros, se agrupan en: a) literarios: cuentos, mitos, fábulas, etc. ; b) recreativos: canciones de cuna, adivinanzas, juegos varios, etc. ; y, c) instructivos: manuales para la enseñanza del quechua, recetas de cocina, pautas para la preparación de potajes y productos artesanales, etc. El conjunto de estos recursos ha sido alojado provisionalmente en el OneDrive-Universidad Nacional Federico Villarreal.

Entre los instrumentos científicos utilizados en la recolección de datos de la Internet figura una ficha para precisar los datos relevantes de cada texto: la base datos o red social de origen, tipo, género y autor, entre otros. Entre los que permitió la reunión de textos creados y recreados, una ficha sociolingüística para identificar a los colaboradores: nombre, sexo, edad, procedencia geográfica, variedad de quechua que maneja, talento lingüístico o cultural específico. Finalmente, una guía práctica con criterios sociales, culturales y pedagógicos para la selección de textos que servirán como contenidos de los materiales de apoyo.

\subsection{Procedimientos:}

En el desarrollo de la investigación fueron empleados los métodos de gabinete, de campo y analítico. El primer método se utilizó en diferentes etapas: al inicio del estudio para la búsqueda de información teórica producida el ámbito latinoamericano (México, Bolivia y Perú) y para la identificación de materiales existentes en la Internet; de forma paralela a la recolección de datos, para la transcripción de material lingüístico; y, culminada dicha tarea, para el análisis, selección, adecuación de los textos escritos y fonográficos acopiados; así como para proponer los criterios básicos que guiarán la elaboración de los materiales didácticos de apoyo para los beneficiarios de los programas de revitalización. El segundo método se

4 Las restricciones establecidas por el gobierno para contener la propagación de la COVID-19 limitaron el desarrollo del trabajo de campo con los beneficiarios directos de los programas de revitalización lingüística. Por tal motivo, la Internet se convirtió en un medio de uso obligatorio. 
Documentación lingüística-cultural y producción de prototipos de material... Carmela Florencia López Capillo, Yris Barraza de García y Rosalinn Cancino Verde

aplicó a través de asistentes comunitarios. Este procedimiento permitió reunir cuentos e historias, orales y escritos. El proceso continuará en los asentamientos humanos de Los Olivos culminada la pandemia causada por la covid-19.

\section{Resultados}

\subsection{Discursos que expresan saberes lingüísticos y culturales}

Los textos que revelan los saberes tradicionales de los pueblos quechua, comunidades a las que pertenecen los migrantes que viven en Lima Metropolitana, son incalculables. A continuación, muestras de los discursos referidos, presentados según los sitios de Internet que los aloja y los géneros indicados en 3.2:

\subsubsection{Materiales recuperados de las redes sociales de entidades públicas, colectivos culturales o personas particulares}

\subsubsection{Género literario}

\section{A. Cuentos y diálogos en formato de video}

Atoqwan kunturwan chiripi llallinakushanku «El zorro y el cóndor. Un reto de altura», en quechua cuzqueño. Producido el 2014, con base en un cuento andino, por Fernando Tagle y el C.E.C. Guamán Poma de Ayala. Compartido en: https://youtu.be/mjlxvurgple.

El sapo y la luna (saludos), en quechua ayacuchano. Producido el 2018 por «Oso con lentes producciones», bajo el título de El sapito vanidoso. Compartido en: https://youtu.be/jgddadcjstg

Kimsa masikunamanta «El cuento de los tres amigos», en quechua boliviano (para niños de familias cusqueñas y puneñas). Relata las reacciones de una oveja, una llama y un perro ante la presencia de un vehículo. Producido el 2019 por «Boliviamanta Qhichwa Misk'i Simi» y compartido a través de: https://youtu.be/RgJMP2flJxU. 
Documentación lingüística-cultural y producción de prototipos de material... Carmela Florencia López Capillo, Yris Barraza de García y Rosalinn Cancino Verde

\subsubsection{Género recreativo}

\section{A. Canciones infantiles en formato impreso}

Ñuqa wirpuy waqaychata yachani «Yo sé cuidar mi cuerpo». Ministerio de Educación. Compartido a través de: www.minedu.gob.pe.

Puñuy wawita «Duerme mi hijita». Creación: Graciela Mendoza. Compartido a través de https://www.youtube.com/watch?v=b3mvvnr5rym.

\section{B. Canciones infantiles en formato de video}

Canción quechua cantada por niñas del Centro de Educación Inicial ЕІв de Pucurhuay, Curpahuasi, Grau, Apurímac. En: https://youtu.be/ kizpP2GIzoI.

Calabacitay: «Mi calabacita», en quechua ayacuchano. Video dirigido y compartido por Andy Chacón AJC en: https://youtu.be/kizPP2GIzoI.

Qam hina: "Como tú». Video realizado por Renata Flores en quechua ayacuchano. Compartido el 2019 en: https://youtu.be/ugkyv2g7hge

\subsubsection{Entre los materiales inéditos alojados en el OneDrive-Universidad Nacional Federico Villarreal, figuran}

\subsubsection{Género literario:}

\section{A. Relatos para niños y jóvenes en quechua de Conchucos Norte}

Fábulas (aranwaykuna): Mishiwan ukush «El gato y el ratón», Ñuyaashuwan chukllush «La hormiga y el grillo», escritas por el profesor Helí Vidal López.

Cuento: Wayrawan tayta achay «El viento y el padre sol», escrito por el profesor Helí Vidal López. 
Documentación lingüística-cultural y producción de prototipos de material... Carmela Florencia López Capillo, Yris Barraza de García y Rosalinn Cancino Verde

Mitos: Pantashqa pishtaqpa wañukuynin «La muerte del pishtaco confundido» y Alfalfeeruwan pishtaq «El alfalfero y el pishtaco», narrado por Luisa Minas García.

\subsubsection{Género recreativo}

A. Canciones de cuna e infantiles

En quechua del Alto Marañón (Huánuco): Punukushuna wawa «iVamos a dormir bebé!», Aku mitsikojkuna: «Vamos pastores!» y Akula niña aywakulashun «iVamos niña, vámonos!», escritas por Elva Verde; y, Piray taqay wamra «¿Quién es aquella niña?», escrita por Marina Verde.

En quechua cusqueño: Allinta mikhunapaq «Para comer bien», Kuntur Phalarin «El Condor Pasa» y Pukay panti pariwana «Parihuana de rojo encendido», traducidas o adecuadas por Fanel Guevara Guevara.

\section{B. Adivinanzas}

En quechua Huaylas: Biyulin «El violín», Sapatu «El zapato», Manka «La olla» y Nina «La candela»; Anta kasha: «La turuna 'espina'», Yukis «El zorzal», Mangu «El mango»; Wishlla: «El cucharón», Rumi mulinu «El molino de piedra», Kamsta «La cancha». Documentadas por Félix Julca Guerrero.

En quechua chanka: Biyulin: «El violín», Sapatu «El zapato», Manka «La olla» y Nina «La candela»; Kichka: «La espina», kuwach «El zorzal», Mangu «El mango»; Wishlla: «El cucharón», Rumi mulinu «El molino de piedra», Hamka «La cancha». Adecuadas por Fernando García Rivera a partir de la versión huaylina.

En quechua Conchucos Norte: Mayu: «El río», Kishiw «La caigua serrana», Tarwi «El chocho», Tukru «El bastón», Pikuyllu «La hierba del maizal», Naqtsa «El peine», Wachuku «El fajín», Kuchara «La cuchara»; Illaqu «El rayo», Pampa killa «La luna llena», Qallu «La lengua»; Kampana «La campana», Kampana «La campana (otra versión)», Putska «El hilado»; 
Documentación lingüística-cultural y producción de prototipos de material... Carmela Florencia López Capillo, Yris Barraza de García y Rosalinn Cancino Verde

Biyulin «El violín», Llantu «La sombra». Documentadas por Helí Vidal López y Aída López Capillo.

\subsubsection{Género instructivo}

\section{A. Instrucciones para tareas gastronómicas}

Haka pichuy «Picante de cuy», Tanta taqllayo Tanta lluylluyo Masarakuy «Preparación de panes y tortas», Asway «La chicha»; Chicharrukuy «El chicharrón», entre otras recetas.

\section{B. Instrucciones para juegos infantiles y juveniles}

Chanta «mundo»; Chunkay «juego de yaces», Boola chunkay «juego con caninas», Pushku chunkay «chocolateo y lanzamiento de frijoles»; Manka chasun «la olla se rebalsa», Trompu hitay «baile del trompo»; entre otros entretenimientos.

\section{Instrucciones para actividades artesanales}

Tsuku ruray «fabricación de sombrero», Pustkay «hilado», Punchu ayaway «Tejido de poncho»; Baata borday «bordado de batas», Manka ruray «fabricación de ollas de barro».

\section{Materiales para actividades formales}

Guías para los usuarios de los programas de retención y de fortalecimiento de la lengua quechua en el distrito de Los Olivos - Lima: Elaboración: Yris Barraza de la Cruz y Carmela López Capillo. Universidad Nacional Federico Villarreal: Laboratorio de Fonética. Lima 2020.

Manual de Enseñanza Quechua: Qichwa simita yachakusun BÁsıco 1. Elaboración: Fernando Antonio García Rivera e Yris Barraza de la Cruz. Universidad Nacional Federico Villarreal: Laboratorio de Fonética

- Centro de Estudiantes. Lima 2015. 
Documentación lingüística-cultural y producción de prototipos de material... Carmela Florencia López Capillo, Yris Barraza de García y Rosalinn Cancino Verde

Entre los discursos seleccionados para la elaboración de materiales de apoyo como: cancioneros, libros de cuentos, libros de adivinanzas, anecdotarios, historietas o materiales de otro tipo, para los programas de revitalización del quechua en el distrito de Los Olivos, figuran:

\subsubsection{Género literario}

\section{A. Fábulas en quechua de Conchucos Norte (Ancash):}

\section{Nuyaashuwan chukllush}

Huk chukllushshi llapan paqas qutsuyllata yachaq, hunaqpanash mallaqar llakikuyta tariq.

- Mayparaq aywaa nishpa. Yarpachakuykanqanpitashi yanasan ñuyaashuman mañakuq aywanaq:

-Tiyay, tiyay ichik haraykita llamiykatsimay niptinnash. ñuyaashuqa yaskiriq:

-Kayllam kan, nishpa makyanaq.

Huk hunaqnash tsaynawlla kutinaq; punkullapitanash rimaykukunaq.

- ¿Imanawllanataq tiyay kaykanki?, niptinshi. Nuyaashuqa yaskirinaq:

_¡Yamayllam! ¿qamqa?, niptinshi yaskirinaq:

-Nuqaqa hinallam qishyaykaa,

- Manatsuraq tiyay purash hara-

llata llakipaykamankiman, niptinshi.

Nuyaashuqa yaskirinaq:

- Allaapa qilatanam waatarquq, paqaskuna qutsuskir nuqallaman yarparaykanki, aywakuy manam rikaynikitapis munatsu, nirshi qarqunaq.

-Chukllushqa, mallaqaypita waqar aywakunaq.

\section{La hormiga y el grillo}

Cuentan que un grillo solía cantar toda la noche y lamentarse durante el día por sentir hambre.

-Decía: ¿A dónde iré?

Un día de tanto pensar resolvió ir donde su amiga la hormiga para pedirle:

-Tía, tía, permíteme arrancar algunas hojas de tu maíz.

La hormiga asintió y le alcanzó diciendo:

- Tengo esto poco nomás para darte.

Otro día regresó con el mismo pretexto y desde la puerta se precipitó a saludar:

- ¿Cómo te encuentras tiíta?

Entonces, la hormiga respondió:

-Yo estoy bien. ¿Y tú?

Al oír a la hormiga, el grillo replicó:

-Yo sigo igual de enfermo.

- No podrías, tiíta, regalarme un puñado de maíz.

La hormiga al escuchar esas palabras lo echó, diciendo:

- He hecho que te acostumbres a estar muy ocioso. Cantas por las noches y luego te confías solo en mí. ¡Vete!, ¡No quiero verte más! 
Documentación lingüística-cultural y producción de prototipos de material... Carmela Florencia López Capillo, Yris Barraza de García y Rosalinn Cancino Verde

Finalmente, el grillo se fue llorando de hambre.

Yachatsikuynin: Ama qilaqa

kawashwantsu, arukuq, yanapakur kawakushwan.
Enseñanza: No debemos ser ociosos; debemos vivir trabajando y ayudando.

Qillqaq: Helí Vidal López.

Selección y versión es español: Carmela López Capillo.

\section{B. Mito en quechua de Conchucos Norte (Ancash):}

\section{Pantakushqa pishtaqpa wañukuynin La muerte del pishtaco que se confundió}

Huk chakwanwan huk awkinshi saachikuyaanaq hallqa chakrankunachaw; tsayshi tsay saachikuykayanqanmanshi huk pishtaq, yaykunaq ishkanta pishtanampaq.

Tsay awkinwan chakwanqa, pishtaq yaykuykanqata, mana musyayantsuqa; tsayshi huknin imata ashiptinchari hukah nin: Hanalaapa hanalaapa, uralaapa uralaapa, kay laapa kay laapa, kay laapa kay laapa. Tsayta wiyask

irninshi pishtaaqa ninaq: nuqatanam qatikachaykayaaman, tsaypitash chakwankunaman yaykuykarpis, sanja ruriman heqaskin wañut.
Cuentan que una anciana y un anciano estaban haciendo el saachi en sus chacras de la puna. Entonces, cuando ellos estaban en esta tarea, un pishtaco entró al lugar para matar a ambos.

Los ancianos desconocían que el pishtaco estaba allí; entonces, quizás porque uno de ellos buscaba algo, el otro le decía: Hanalaapa hanalaapa (por arriba por arriba); uralaapa uralaapa (por abajo, por abajo); kay laapa kay laapa (por aquí, por aquí). Al oír eso, el pishtaco pensó que lo estaban persiguiendo; $y$, aunque estuvo entrando para matarlos, cayó a una zanja profunda y se murió.

*Saachi: práctica de abonar la tierra con el guano que excretan las ovejas y cabras. Narración: Luisa Minas García.

Adaptación y traducción: Carmela López Capillo. 
Documentación lingüística-cultural y producción de prototipos de material... Carmela Florencia López Capillo, Yris Barraza de García y Rosalinn Cancino Verde

\subsubsection{Género recreativo:}

\section{A. Ronda de cuna en quechua del Alto Marañón (Huánuco)}

\section{Punukushuna wawa}

Punuy puny wamra; punuy punuy niña.

Tsakay yaykaykamunnami, aku punukushunna.

Punuy puny wamra; punuy punuy niña. Takay sellamami qasan; sumaj pitukushun hakuwan; sumaj pitukushun kaamawan, mana qasanantsipa.

Punuy puny wamra; punuy punuy niiña.

\section{¡Vamos a dormir, bebé!}

Duerme; duerme, niño. Duerme; duerme, niña. La noche ya se acerca: ¡Vamos a dormir! Duerme; duerme, niño. Duerme; duerme, niña. La noche es muy fría; envolvámonos suavemente con la manta; envolvámonos suavemente con la cama, para no sentir frío. Duerme; duerme, niño; Duerme; duerme, niña.

Creación: Elva Verde.

Documentación, selección y adecuación: Rosalinn Cancino Verde.

\section{B. Canción popular en quechua huanuqueño}

\section{Akula niña awakulashun}

Akula niña aywakulashun Taqay punta wajtalaman Mana pipis rikamashun, Akula niña aywakulashun, taqay punta wajtalaman, mana pipis rikamasun. Akula niña aywakulashun, taqay punta wajtalanman kondorkuna takunman.

\section{¡Vamos, niña; vayámonosi}

¡Vamos, niña; vamos, hacia la espalda de aquella cordillera, donde nadie nos encuentre! ¡Vamos niña; vamos, hacia la espalda de aquella cordillera, donde nadie pueda hablar sobre nosotros! ¡Vamos niña, vámonos, hacia la espalda de aquella cordillera donde los cóndores viven!

Creación: Marina Verde.

Documentación, selección y adecuación: Rosalinn Cancino Verde. 
Documentación lingüística-cultural y producción de prototipos de material... Carmela Florencia López Capillo, Yris Barraza de García y Rosalinn Cancino Verde

\section{Canción para niños en quechua cusqueño}

\author{
Allinta mikhunapaq \\ Huwina sarachawan, \\ paraqay sarachawan, \\ chakramanta huqarikusunchis \\ allinchata mikhukunanchispaq \\ Huch'uycha papachata, q'umircha \\ habaschata, chakramanta huqarikusun- \\ chis allinchata mikhukunanchispaq.
}

Quwita kankasunchis, mut'ita wayq'usunchis; allinchata mikhurukusunchiq allinchata kausakunanchispaq.

\section{Para comer bien}

Con el maíz amarillo, con el maicito blanco, recogeremos de nuestra chacra para que comamos bien.

Las papitas chiquitas, las habitas verdecitas, recogeremos de nuestra chacra para que comamos bien.

Creación y versión en español de Fanel Guevara Guevara.

\subsection{Características de los prototipos de materiales de apoyo para la ejecución de programas de revitalización lingüística}

Criterios establecidos para la producción de materiales didácticos para el desarrollo de actividades académicas con estudiantes del sistema escolar regular rigen también la construcción de materiales de apoyo para la revitalización lingüística; sin embargo, para que estos contribuyan con eficacia al esfuerzo de los migrantes quechua hablantes en la tarea de restablecer la línea de la transferencia intergeneracional de la lengua nativa actualmente rota en los asentamientos humanos de Lima, estos deben considerar las siguientes características:

\subsubsection{En cuanto a los contenidos}

Mostrar con respeto y creatividad una amplia variedad de saberes tradicionales con los que sí cuentan los pueblos quechuas. Sin embargo, para responder a las necesidades comunicativas actuales de los hablantes deben incorporar también conocimientos acerca de la vida en la ciudad y en el mundo moderno. Con este propósito, corresponde que sean renovados periódicamente. Finalmente, los 
Documentación lingüística-cultural y producción de prototipos de material... Carmela Florencia López Capillo, Yris Barraza de García y Rosalinn Cancino Verde

contenidos deben ser tratados con criterios éticos y en sintonía con criterios psicopedagógicos como la selección y la dosificación de la información, según el programa objetivo: retención, fortalecimiento o aprendizaje y las edades de los participantes, la coherencia y la cohesión textual.

\subsubsection{En cuanto a la estructura}

Todos deben ser simples, sencillos y atractivos; especialmente, aquellos que son destinados para el trabajo con los niños y los adolescentes. En tal sentido, las especies literarias, recreativas e instructivas que, -debido a la simplicidad de sus estructuras y a la facilidad con que pueden ser presentados bajo distintos formatos--, favorecen la interacción con los grupos etarios mencionados son los cuentos breves, los relatos cortos, las fábulas, las canciones, las adivinanzas y los juegos. Por lo demás, la distribución de los textos al interior de los materiales debe atender las recomendaciones de maestros y diseñadores especializados en la producción de materiales educativos; los diseños de los códigos visuales (letras, gráficos e imágenes), orales (sonidos) y audiovisuales (sonidos e imágenes en movimiento) deben sintonizar con la manifestación organizada, clara y entretenida de los contenidos.

\subsubsection{En cuanto a la funcionalidad}

En conjunto, se requiere materiales fáciles de utilizar y con las dimensiones y los pesos adecuados para su portabilidad. Los que han de ser fabricados en soportes físicos (libros, fichas, cartillas, tableros y otros) deben ser confeccionados con materia prima de óptima calidad (principalmente, no tóxica y de origen peruano) para cuidar la salud de los usuarios, así como la duración y la reusabilidad de los productos. En la misma línea, los materiales, especialmente los digitales, deben facilitar la interacción: adulto - niño; orientador - orientado, o la exploración y el manejo autónomos de los participantes en el programa de revitalización. En todo momento, se debe considerar que los objetivos son concretos: la transferencia - adquisición, el fortalecimiento o la enseñanza - aprendizaje de la lengua quechua. Para contribuir al logro de los propósitos, cada ejemplar debe incluir un instructivo fácil de comprender. 
Documentación lingüística-cultural y producción de prototipos de material... Carmela Florencia López Capillo, Yris Barraza de García y Rosalinn Cancino Verde

\subsubsection{Considerando los programas y sus beneficiarios}

Se debe prever materiales para los programas de retención, fortalecimiento y aprendizaje con las características de contenido, estructura y funcionalidad correspondientes. Los que han de ser útiles en el trabajo presencial extraescolar y a distancia con niños que asisten a escuelas primarias también deben tener las cualidades establecidas para los materiales de revitalización desde los hogares y desde los espacios comunitarios no formales. Esto porque el programa de revitalización considera también talleres que forman parte de un aprendizaje formal de la lengua para tres grupos diferentes: niños muy pequeños que asisten a wawawasis, nidos, o centros de educación inicial; niños más grandecitos que participen en la escuela primaria; y, jóvenes adolescentes que estudien en colegios secundarios o centros de educación superior. La revitalización de la lengua en estos espacios requiere de la participación de una madre de familia (Clemente, 1998) y/o de una maestra o maestro quechua hablante; para ellos, se plantea: una guía de uso de la lengua para la motivación y formas de interacción con los niños y ayudas visuales en formato PPT sobre experiencias de aprendizaje de lenguas en contextos similares. Para el aprendizaje del quechua como segunda lengua se adecuará el manual producido en quechua ayacuchano por García y Barraza (2015) a otras variedades.

\section{Discusión}

\subsection{Con relación a los resultados 5.1}

La producción verbal relacionada con los saberes lingüísticos y culturales acopiada hasta la actualidad es abundante, de diverso género y de variada extensión. Los discursos tienen como principal destino la construcción de materiales de apoyo para la revitalización de la lengua quechua en un espacio geográfico y social específico; sin embargo, debido a que son productos de una actividad realizada por miembros de una entidad universitaria, constituirán un acervo disponible para la investigación científica. Culminadas, la organización de los datos y la implementación del repositorio institucional, lingüistas, literatos, antropólogos, sociólogos y profesionales de otras especialidades podrán acceder a la totalidad de testimonios lingüísticos. De esta forma, la documentación lingüística habrá cumplido con los dos objetivos que Himmelman (1998 y 2007) propone para los datos primarios que resulten de pesquisas científicas: servir a la comunidad de hablantes de la lengua documentada y a las comunidades académicas interesadas.

118 Lengua y Sociedad. Revista de Lingüística Teórica y Aplicada 
Documentación lingüística-cultural y producción de prototipos de material... Carmela Florencia López Capillo, Yris Barraza de García y Rosalinn Cancino Verde

\subsection{Con relación a los resultados 5.2}

Austin (2007) señala que el conjunto de datos útiles para el mantenimiento y el aprendizaje de una lengua amenazada debe ser diverso, amplio, continuo, distribuido y flexible; transparente, preservable, portátil y ético. Los datos en lengua quechua recogidos reúnen los requisitos que el autor propone. Corresponden a diversos géneros y subgéneros literarios, recreativos e instructivos. Los relatos, las fábulas, los mitos, los refranes, las adivinanzas; las rondas, las canciones de cuna, las canciones del folclor popular; los instructivos para la realización de los juegos, la preparación de alimentos, la elaboración de artesanías; entre otros datos primarios, son abundantes e inagotables; por tanto, la amplitud, la continuidad y la flexibilidad de los datos están garantizadas. Zariquey y otros (2019), López (2015), Flores et al., 2020, INALI (2012), sin embargo, afirman que la reunión de datos primarios y la creación de materiales, requiere trabajos colaborativos entre: Estado - comunidad - academia - empresa y otros agentes de la sociedad. Finalmente, López y Barraza (2018) recomiendan la previsión de información útil para la sensibilización permanente de los padres y demás miembros de las familias migrantes.

\subsection{Con relación a los resultados $\mathbf{5 . 3}$}

La tarea de documentar una lengua amenazada compromete la contribución de todos los actores de la comunidad de hablantes: padres de familia; dirigentes, autoridades locales, regionales; cultivadores de la lengua (poetas, compositores, intérpretes) y de la música; amautas de la agricultura, la textilería, la cerámica y demás actividades culturales; pues, en conjunto, poseen los saberes de sus pueblos, como los quechua hablantes reúnen los suyos. La construcción de los materiales de apoyo para la revitalización de una lengua amenazada, con las características señaladas en 4.3, demanda también la tarea de profesionales del área de las ciencias humanas: lingüistas, literatos, educadores, antropólogos, actores; ingenieros de sonido y electrónicos, entre otros especialistas. El reto es inmenso (Cru, 2014); en casos como el presente, asegurar los recursos humanos y económicos para implementar un programa de revitalización autosostenible en el tiempo, pondrá a prueba la eficacia de la alianza Estado-comunidad-academia-empresa (López, 2015; Flores et al., 2020; INALI, 2012). 
Documentación lingüística-cultural y producción de prototipos de material...

\section{Conclusiones y recomendaciones}

7.1. Los saberes tradicionales de los quechua hablantes de todas las variedades son abundantes, de variado género y de riqueza incalculable. En tal sentido, la existencia de textos literarios, recreativos e instructivos de todas las especies, realidad que -actualmente- es posible conocer y valorar a través de la Internet, demuestran la vitalidad de la lengua en las comunidades de origen de los migrantes. Por las razones señaladas, la gestión de programas para la revitalización de la lengua quechua en contextos urbanos debe considerar la participación de miembros de las comunidades lingüística de procedencia de los beneficiarios.

7.2 Entre los materiales reunidos (y entre aquellos otros que se encuentran en fuentes orales, gráficas y electrónicas) hay numerosos cuentos, narraciones, canciones y recetas culinarias quechuas de todas las especies y, en menor cantidad, adivinanzas, refranes, metáforas e instrucciones para juegos; adecuados, interesantes y atractivos para su empleo directo o para la elaboración de materiales de apoyo para los programas de revitalización de la lengua nativa en contextos urbanos. Una estrategia lingüística interesante para lograr revitalizar una lengua polidialectal como el quechua es la creación de textos en una variedad quechua y su adaptación a otras variedades. Esta estrategia lingüística pretende fomentar la práctica de la interculturalidad entre los miembros de los diferentes pueblos quechua asentados en el distro limeño de Los Olivos; no obstante, la experiencia deberá ser valorada en función a sus resultados. Esto para su replicación en realidades similares.

7.3 Los materiales que se produzcan para apoyar las actividades de la revitalización de la lengua quechua en los asentamientos humanos del distrito de Los Olivos deben reunir todas las características que distinguen a los buenos materiales que son utilizados en la educación básica y en la enseñanza de segundas lenguas. Sin embargo, para que estas herramientas sirvan realmente a los propósitos de la retención lingüística en un contexto tan difícil y extremadamente homogeneizador como el que tipifica a Lima, deben ser muy atractivas; especialmente para los niños y los adolescentes. Esto implica que deben presentar los saberes culturales con mucha creatividad, los tradicionales y también los de la vida moderna; asimismo, los formatos elegidos deben ser funcionales, durables y fáciles de transportar para todos. Finalmente, producir materiales con las bondades señaladas demanda no pocos recursos humanos y económicos; por tanto, alcanzar el reto dependerá también del respaldo de las entidades gubernamentales, de la adhesión de otras universidades y de la contribución de las empresas privadas. 
Documentación lingüística-cultural y producción de prototipos de material... Carmela Florencia López Capillo, Yris Barraza de García y Rosalinn Cancino Verde

\section{Referencias Bibliográficas}

Austin, P. (2007). Los datos y la documentación lingüística. En J. Gippert y N. Himmelmann y M. Ulrike (Eds.), Essentials of Language Documentation [versión en español: Bases de la documentación lingüística] (pp. 111-140). Mouton de Gruyter.

Cru, J. (2014). Loreword. En J. Cru (Coord.), The Management of Multilingualism in Urban Settings (pp. 4-6). Linguapax Review.

Clemente, R. (1998). El papel del adulto en la adquisición del lenguaje. Reflexiones sobre los valores diferenciales entre interlocutores. En Peralbo, M., Gómez, B., Santorúm, R. y García, M., Desarrollo del lenguaje y cognición (pp. 41-51). Pirámide.

Decreto N. ${ }^{\circ}$ 004-2016-MC. Decreto Supremo que aprueba el Reglamento de la Ley $\mathrm{N}^{\circ} 29735.22$ de julio de 2016. D. O. N. ${ }^{\circ} 593850$.

Flores, J. A., Córdova, L. y Cru, J. (2020). Guía de revitalización lingüística: para una gestión formada e informada. Linguapax International. http://www.uabjo. $\mathrm{mx} / \mathrm{media} / 1 / 2020 / 06 /$ GuiadeRevitalizacionLinguaPax-UABJo.pdf

García, F. y Barraza, Y. (2015). Qichwa simita yachakusun. Básico 1. Universidad Nacional Federico Villarreal: Laboratorio de Fonética - Centro de Estudiantes de Lingüística - Instituto de Investigación Facultad de Humanidades.

Gutiérrez, R. (2013). La dimensión lingüística de las migraciones internacionales. Lengua y Migración, 5(2), 15-28. https://www.redalyc.org/articulo. oa? id $=519551815002$

Himmelmann, N. (2007). La documentación lingüística: ¿qué es y para qué sirve? En J. Gippert, N. Himmelmann y M. Ulrike (Eds.), Essentials of Language Documentation (pp. 15-47). Mouton de Gruyter. [versión en español: Bases de la documentación lingüística. Instituto Nacional de Lenguas Indígenas]

Himmelmann, N. (1998). Documentary and Descriptive Linguistics. Linguistics, 36, 161-195.

Instituto Nacional de Lenguas Indígenas [INALI]. (2012). Documentación Lingüística. Memoria Documental: Informe de Rendición de Cuentas (20062012). https://site.inali.gob.mx/pdf/documentacion_linguistica.pdf

Instituto Nacional de Estadística e Informática [INEI]. (2018). Censos Nacionales: XII de Población, VIII de Vivienda y I de Autoidentificación. https://www.inei. gob.pe/media/menurecursivo/publicaciones_digitales/est/lib1539/libro.pdf 
Documentación lingüística-cultural y producción de prototipos de material...

Carmela Florencia López Capillo, Yris Barraza de García y Rosalinn Cancino Verde

Ley 29735 de 2011. Ley que regula el uso, preservación, desarrollo, fomento y difusión de las lenguas originarias de Perú. 5 de julio de 2011. D. O. N. ${ }^{\circ} 445799$.

Ley 30220 de 2014. Ley Universitaria. 3 de julio de 2014. D. O. N. ${ }^{\circ} 527211$.

Leyva, A. (2014). Documentando una lengua: El caso kumiai. Revista Digital Universitaria, 2(15), 1-10. http://www.revista.unam.mx/vol.15/num2/art11

López, C., Sullcahuamán, A. y Barraza, Y. (2019). Migración, variación dialectal $y$ transferencia intergeneracional de la lengua quechua en familias migrantes de los asentamientos humanos Enrique Milla Ochoa, Laura Caller y Armando Villanueva del distrito de los Olivos - Lima (Informe final de investigación). Universidad Nacional Federico Villarreal, Lima.

López, C. y Barraza, Y. (2018). Transferencia intergeneracional de la lengua quechua en familias migrantes de los asentamientos humanos Enrique Milla Ochoa, Laura Caller y Armando Villanueva del distrito de los Olivos - Lima. En A. Regúnaga, S. Spinelli y M. E. Orden (Comps.), IV Encuentro de Lenguas Indígenas Americanas (eLIA). Libro de Actas (pp. 427-448). UNALPam. http:// www.unlpam.edu.ar/images/extension/edunlpam/actas\%2oelia\%20-\%20 unlpam.pdf

López, L. E. (2015). El hogar, la comunidad y la escuela en la revitalización de las lenguas originarias de América. En L. López (Ed.), Pueblos Indígenas y Educación N. 64. Abya-Yala. https://www.academia.edu/31014392/el_hogar_ la_comunidad_y_la_escuela_en_la_revitalizaci\%c3\%b3n_ling\%c3\%bcistica

Organización Internacional para las Migraciones [OIM]. (2015). Las migraciones internas en el Perú en su contexto actual. http://www.oimperu.org/sitehome/ sites/default/files/documentos/03-03-2015_publicacion\%2omigraciones\%20 internas_oim.pdf

Pontificia Universidad Católica del Perú (s. f.). Archivo Digital de Lenguas Peruanas [Página web]. http://repositorio.pucp.edu.pe/index/handle/123456789/124179

Rodríguez, G. y Magaña, J. (2017). Revitalización de la lengua y la cultura a través de la música. Boletín Antropológico, 35(94). https://www.redalyc.org/ jatsrepo/712/71256055006/71256055006.pdf

Unesco. (2003). Vitalidad y peligro de desaparición de las lenguas [Documento]. http://www.unesco.org/new/fileadmin/multimedia/hq/clt/pdf/lve_spanish_ edited\%2ofor\%2opublication.pdf

Valqui, J., Cosar, R., Jimenez, P., Ramos, L., Suclupe, P. y Villanueva, B. (2016). La documentación lingüística en la investigación de lenguas en la Amazonía 
Documentación lingüística-cultural y producción de prototipos de material... Carmela Florencia López Capillo, Yris Barraza de García y Rosalinn Cancino Verde

peruana. Lengua y Sociedad, 14(2), 62-77. http://revista.letras.unmsm.edu.pe/ index.php/ls/article/view/319/314

Zariquiey, R., Hammarström, H., Arakaki, M., Oncevay, A., Miller, J., García, A. y Adriano Ingunza (2019). Obsolescencia lingüística, descripción gramatical y documentación de lenguas en el Perú: hacia un estado de la cuestión. Lexis, 43(2), 271-337.

\section{Trayectoria académica de las autoras}

\section{Carmela Florencia López Capillo}

Docente de la Especialidad de Lingüística en la Universidad Nacional Federico Villarreal (UNFV) desde 1988. Tiene estudios de pregrado en Educación (UNFV) y de pregrado, diplomado, maestría y doctorado en Lingüística (UNMSM). Investiga sobre temas de Lingüística Quechua, Psicolingüística y Sociolingüística desde 1997. Entre los trabajos de investigación que ha publicado figuran: Transferencia intergeneracional de la lengua quechua en familias migrantes de los asentamientos humanos Enrique Milla Ochoa, Laura Caller y Armando Villanueva del distrio de Los Olivos-Lima (UNALP-Argentina), Hacia una escritura del quechua ancashino (Revista Tipshe-Unfv). Es miembro de la Sociedad de Lingüistas del Perú, la Red de Egresados de la Universidad Intercultural Indígena (Red uiI) y la Red de Gestores de Investigación del Perú. Por su experiencia en la producción de conocimientos científicos y en la gestión de la investigación desarrolla cursos formativos en estos campos; asimismo, asesora tesis y trabajos de investigación de estudiantes de las carreras del área de las Humanidades. Se ha desempeñado como jefa de la Oficina de Evaluación Científica (2011-2013), directora del Instituto de Investigación de la Facultad de Humanidades, en dos oportunidades (2004-2007 y 2014-2016) y directora de la Escuela Profesional de Lingüística y Literatura de la institución donde trabaja (2001-2004). También se ha desempeñado como Especialista en Educación Intercultural y Bilingüe en el Ministerio de Educación (1992-1995). 
Documentación lingüística-cultural y producción de prototipos de material... Carmela Florencia López Capillo, Yris Barraza de García y Rosalinn Cancino Verde

\section{Yris Barraza de García}

Es doctora en Letras (UFP), magíster en Lingüística (UNMSM), responsable del Área de Comunicación e Investigadora de la lengua Shawi en el Programa de Formabiap.

\section{Rosalinn Cancino Verde}

Es magíster en Lingüística (UNMSM), licenciada en Lingüística (UNFV), estudios de doctorado en curso en Lingüística (UNMSM) y docente de la UnFv. Trabajó como docente de taller de lenguas peruanas en la UCH, y en la Escuela de Inteligencia del Ejército Peruano como docente en quechua. Ha publicado El quechua de Santa Rosa de Patopampa: la despalatalización (libro de investigación), «Etnotaxonomía de algunas aves nocturnas en el quechua de La Unión» (Revista de investigación Tesis, UnMsM), coautora en el artículo «El mapa mental armónico en la comprensión de textos narrativos en estudiantes universitarios» (revista UsIL. Propósitos y Representaciones), coautora en «El aprendizaje ubicó en el proceso de enseñanza aprendizaje» (revista Multi_Ensayos), Denominación de nombres de aves en el quechua de La Unión - Huánuco (Cybertesis, UnMSM), «Hispanismos en el quechua de Humalíes-Huánuco» (II Congreso Histórico de Investigaciones HistóricoSociales de la Región Huánuco: LLata) entre otras investigaciones en revistas indexadas. Participó como conferencista en eventos internacionales y nacionales.

124 Lengua y Sociedad. Revista de Lingüística Teórica y Aplicada 